\title{
Nutrition Outweighs Defense: Myzus persicae (Green Peach Aphid) Prefers and Performs Better on Young Leaves of \\ Cabbage
}

\author{
He-He Cao ${ }^{1,2}$, Zhan-Feng Zhang ${ }^{1}$, Xiao-Feng Wang ${ }^{2}$ and Tong-Xian Liu ${ }^{1 *}$ \\ ${ }^{1}$ Key Laboratory of Northwest Loess Plateau Crop Pest Management of Ministry of \\ Agriculture, College of Plant Protection, Northwest A\&F University, Yangling, \\ Shaanxi, 712100, China, \\ ${ }^{2}$ College of Horticulture, Northwest A\&F University, Yangling, Shaanxi, 712100, \\ China
}

Running title: Leaf ontogeny affects aphid performance

*Correspondence: Tong-Xian Liu

E-mail: txliu@nwsuaf.edu.cn

Keywords: phloem sap, source-sink, electrical penetration graph (EPG), amino acids, glucosinolates, leaf ontogeny

E-mails:

H.H. Cao: caohehe1988@163.com

Z.F. Zhang:25528564@qq.com

X.F. Wang: wangxff99@nwsuaf.edu.cn 


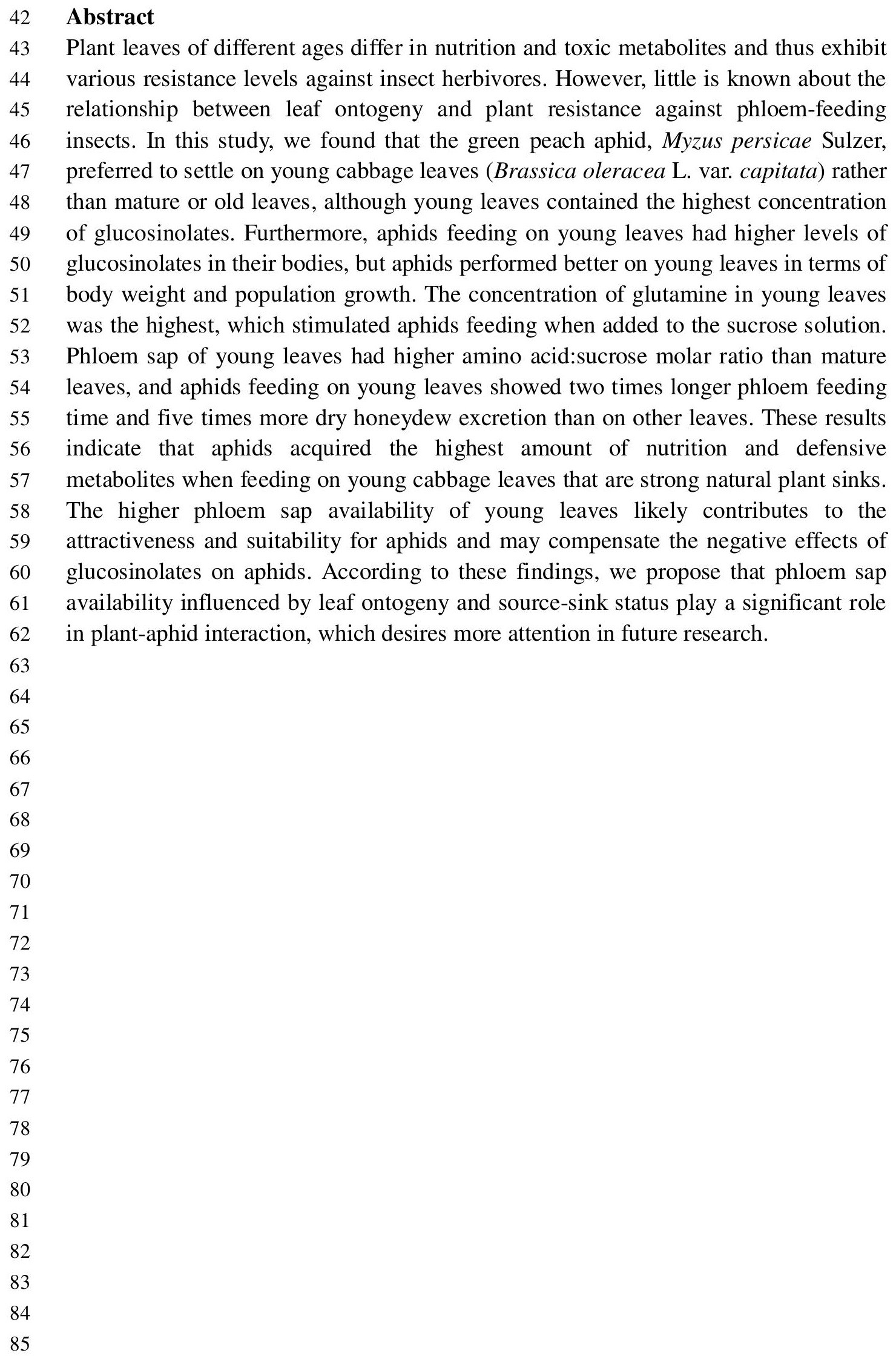




\section{INTRODUCTION}

The optimal-defense hypothesis suggests that valuable tissues of a plant should be better defended against insect herbivores (Barton and Koricheva, 2010). Young leaves of plants usually have higher growth capacity and are more valuable than older ones; therefore, young leaves generally contain more defensive metabolites than older leaves (Schuman and Baldwin, 2016). Thus, insect herbivores are expected to prefer older leaves to minimize the plant resistance conferred by defensive metabolites. However, many insects still prefer and grow better on young leaves, which generally contain more nutrition, suggesting that insects have evolved strategies to minimize the negative impacts imposed by defensive metabolites (Barton and Koricheva, 2010; Kohler et al., 2015). Some studies have examined the relationship between leaf ontogeny and plant defense against the insects with chewing mouthparts, but little is known about leaf ontogeny on plant resistance to phloem-feeding insects (Barton and Koricheva, 2010; Schuman and Baldwin, 2016).

Aphids mainly feed from phloem sap of their host plants, which contains a large amount of sucrose and few essential amino acids, providing an unbalanced diet (Douglas, 2003). During their long period of coevolution, aphids have evolved strategies to cope with these constraints by increasing amino acids in the phloem and harboring symbiotic bacteria to synthesize essential amino acids (Douglas, 2003; Cao et al., 2016). Some aphids can create strong sinks at the feeding sites, increasing the flow of nutrients to the infested tissues and thereby enhancing the availability and nutrition quality of the phloem sap (Larson and Whitham, 1991; Girousse et al., 2005; Züst and Agrawal, 2016). In addition, like other feeding style insect herbivores, aphids have evolved behavioral strategies to increase their fitness by choosing particular host plants and feeding sites within them (Powell et al., 2006). Free-living aphids commonly feed on plant active sinks, like young leaves and reproductive tissues where phloem sap flows to, whereas these plant tissues generally contain more defensive metabolites (Gould et al., 2007). Therefore, there seems to be a conflict for aphids between maximizing nutrition and minimizing exposure to toxic metabolites.

Glucosinolates are the major defensive metabolites in Brassica plants, conferring resistance to most of the insects (Hopkins et al., 2009). Intact glucosinolates can be toxic to insects and their toxic effects are enhanced after hydrolysis by the enzyme myrosinase following tissue damage (Hopkins et al., 2009). Since aphids feed from phloem sap using their slender stylets, they cause tiny tissue damage to their host plants and so rarely contact these toxic breakdown products during feeding. However, indole glucosinolates have a strong antifeedant effects on the green peach aphid, Myzus persicae Sulzer, even without contacting myrosinase (Kim et al., 2008). Despite the negative effects of glucosinolates on aphids, $M$. persicae can grow better on plants containing higher levels of glucosinolates, suggesting that $M$. persicae may has, at least partially, adapted to these metabolites (Cole, 1997; Cao et al. 2016).

For the phloem-feeding insects, the amino acid in phloem sap is the main nutrition, and the amino acid concentration and composition as well as amino acid:sugar molar ratio in phloem sap are important indicators of nutrition quality for phloem-feeding insects (Douglas, 2003). Young leaves generally have higher phloem nutrition quality 
130 and more defensive metabolites; however, little is known about the relative importance of these two kinds of metabolites in plant resistance against aphids. We previously found that the generalist aphid, $M$. persicae, preferred to settle on young cabbage leaves, Brassica oleracea L. var. capitata. In order to evaluate the relative importance of nutrition and plant defense in $M$. persicae-cabbage interaction, we assessed the preference and performance of $M$. persicae on different-aged leaves of $B$. oleraceae. We also measured the glucosinolates and amino acids in different-aged cabbage leaves. Aphid feeding behavior was monitored and their honeydew excretion rate was quantified. This study intends to assess the relative importance of plant nutrition and plant defense in determining $M$. persicae feeding preference and performance on cabbage leaves.

\section{MATERIALS AND METHODS}

\section{Plants and Aphids}

Cabbage seeds (Brassica oleracea L. var. capitata, var. "Qingan 70") were sown in the ground in a greenhouse $\left(23 \pm 5^{\circ} \mathrm{C}\right)$ under natural light conditions. After two months, similar-sized seedlings were transferred to 10 -cm-diameter pots containing soil mixture (peat moss:perlite $=5: 1$ ) and placed in a growth chamber under a 14:10 h $\mathrm{L} / \mathrm{D}$ cycle at $22 \pm 2^{\circ} \mathrm{C}$ and $50 \%$ relative humidity ( $\left.\mathrm{RH}\right)$. The seedlings were watered with tap water as required and drenched with water-soluble fertilizer (2 g/L, 20-20-20 $+\mathrm{Mg}+$ Trace elements; COMPO Expert $\mathrm{GmbH}$ ) every five days, receiving 5 times fertilization in total. The plants were used approximately one month after transplanting, at which time they had about 11-13 leaves. We defined "young leaves" as newly emerged leaves with a diameter smaller than $3 \mathrm{~cm}$, "mature leaves" as fully expanded leaves (about the sixth to seventh emerged leaves), and "old leaves" as those closest to the soil surface. Myzus persicae were reared on two-month-old cabbage "Qingan 70" in cages for more than one year in the same growth chamber. If not otherwise indicated, adult $M$. persicae with similar size were used in the following experiments.

\section{Leaf Age and Aphid Feeding Preference}

Leaf discs (1 cm diameter) were cut from different-aged cabbage leaves using a steel puncher. Two leaf discs from different age groups (i.e., young vs. mature, young vs. old, and mature vs. old) were placed in one Petri dish (9 $\mathrm{cm}$ diameter) lined with wet filter paper and approximately 13 apterous adult $M$. persicae were introduced to the center of these dishes. The number of aphids that had settled on each leaf disc was counted after 1, 2, 3 and $8 \mathrm{~h}$.

\section{Leaf Age and Aphid Performance}

This assay was performed with intact plants. Three apterous adult $M$. persicae were confined on a young, mature or old leaf using a nylon mesh bag (Cao et al., 2016). The petioles were wrapped with cotton to prevent mechanical damage by the bag. After $24 \mathrm{~h}$, the adult $M$. persicae were removed, leaving five newborn nymphs on 
each leaf. After a further $9 \mathrm{~d}$, the aphids were collected and weighed on a microbalance (resolution $0.001 \mathrm{mg}$; Sartorius MSA 3.6 P-000-DM, Gottingen, Germany), and the numbers of adults and nymphs were counted.

\section{Amino Acid Preferences of Aphids}

Myzus persicae feeding preference for amino acids was assessed using $15 \%$ sucrose solution containing each of the 20 amino acids that makes up protein. Thirty-five microliter of $15 \%$ sucrose solution (control) and $15 \%$ sucrose solution containing 3 $\mathrm{mg} / \mathrm{mL}$ individual amino acid were confined separately between two layers of stretched Parafilm M on a plastic Petri dish (1 cm high, $3 \mathrm{~cm}$ diameter). Then, 12 apterous adult $M$. persicae were introduced to each Petri dish and the number of aphids settled on each solution were recorded every 24 hours, lasting for $3 \mathrm{~d}$.

\section{Amino Acid and Sugar Analysis}

To investigate the relationship between nutrition quality and leaf age, we measured the amino acid contents in different-aged leaves and phloem sap. Amino acids in leaves were extracted by grinding the leaves in $0.05 \mathrm{M} \mathrm{HCl}$ with a glass mortar and pestle and were analyzed using an LTQ XL linear ion trap mass spectrometer (Thermo Fisher Scientific, Waltham, MA, USA) as described previously (Thiele et al., 2008; Cao et al., 2014a). We used an EDTA-facilitated exudate method to collect phloem sap. The petioles of cut leaves were immersed in $800 \mu \mathrm{L}$ of $5 \mathrm{mM}$ EDTA solution (pH 7.0) for $3 \mathrm{~h}$ in a dark growth chamber $\left(22^{\circ} \mathrm{C}, 100 \% \mathrm{RH}\right)$. To determine the amino acid:sugar molar ratio in the phloem, we also analyzed the concentrations of sucrose, glucose, and fructose in the phloem exudate using the LTQ XL linear ion trap mass spectrometer, as previously described (Cao et al., 2016). These sugars are the main carbohydrate in the phloem exudate, while all others sugars were less than $1 \mathrm{mg} / \mathrm{L}$ and were not calculated.

\section{Glucosinolates Extraction and Analysis}

To examine the defensive metabolite content in different-aged leaves, we analyzed the concentrations of glucosinolates in young, mature and old cabbage leaves. To inactivate the myrosinase in the leaves, $100 \mathrm{mg}$ of leaves were placed in a $50-\mathrm{mL}$ centrifuge tube and kept in a $96^{\circ} \mathrm{C}$ water bath for $3 \mathrm{~min}$ (Cao et al., 2016). The leaves were then ground with a glass mortar and pestle in $1 \mathrm{~mL}$ MilliQ water, and the mixture was centrifuged at $12,000 \mathrm{~g}, 4^{\circ} \mathrm{C}$ for $15 \mathrm{~min}$. The supernatant was then collected and filtered through $0.22 \mu \mathrm{m}$ syringe filters. Glucosinolates were analyzed using the LTQ XL linear ion trap mass spectrometer (Thermo Fisher Scientific, Waltham, MA, USA), as described previously (Rochfort et al., 2008; Cao et al., 2016). The relative amounts of glucosinolates were calculated according to a standard curve made by 2-propenyl glucosinolate (sinigrin). We collected aphids from different-aged cabbage leaves and analyzed the glucosinolates in their bodies as described above.

\section{Honeydew Excretion Assay}

To investigate aphid feeding rate on different-aged leaves, we measured the dry 
218 weight of honeydew excreted by aphids feeding on young, mature and old cabbage

219 leaves. Five adult aphids were confined to the abaxial side of each leaf using a clip

220 cage. After $24 \mathrm{~h}$, any nymphs that had been produced were removed and the clip 221 cages were replaced by new clip cages lined with aluminum foil for a further $20 \mathrm{~h}$. These clip cages were placed beneath the aphids so that honeydew that was produced dropped onto the aluminum foil. The aluminum foil was dried to a constant weight in a drying oven at $50^{\circ} \mathrm{C}$ before and after the collection of honeydew and the dry weight of honeydew produced per aphid per hour was calculated.

\section{Aphid Feeding Behavior}

We monitored the feeding behavior of $M$. persicae on young, mature and old leaves using the Giga-8 direct-current electrical penetration graph (DC-EPG) system (W. Fred Tjallingii, Wageningen University, Netherlands). Aphid feeding activities were recorded for $8 \mathrm{~h}$ in a Faraday cage at $24^{\circ} \mathrm{C}$. An $18 \mu \mathrm{m}$ diameter gold wire was attached to the dorsum of each aphid using silver conductive glue and then aphids were placed onto leaf surface. Each adult $M$. persicae and cabbage plants were used only once. Signal was recorded by the Stylet+d software and the EPG waveforms were recognized and labeled using the Stylet+ software according to Prado and Tjallingii (1994). Both software was provided by Prof. Tjallingii (Wageningen University, Netherlands). EPG parameters were calculated using the Excel workbook for automatic parameter calculation of EPG data 4.3 (Sarria et al., 2009).

\section{Callose Assays}

Mixed instar M. persicae (30 mg) were confined on the abaxial side of leaves using clip cages $(1 \mathrm{~cm}$ diameter) for $3 \mathrm{~d}$. As a control, some leaves were caged without aphids for $3 \mathrm{~d}$. Leaves wounded by a needle $(0.2 \mathrm{~mm}$ diameter) for several times were positive controls. Following collection, the leaves were fixed in ethanol:glacial acetic acid (3:1) and shaken overnight (Nishimura et al., 2003). The leaves were then decolorized in $98 \%$ ethanol for $2 \mathrm{~h}$ and in $50 \%$ ethanol for $2 \mathrm{~h}$, washed 3 times in distilled water, and stained with $0.1 \%(\mathrm{w} / \mathrm{v})$ aniline blue in $75 \mathrm{mM}$ phosphate buffer ( $\mathrm{pH}$ 9.5) for $4 \mathrm{~h}$ in the dark. Callose deposits were viewed by a fluorescent microscope (EX 330-380 nm; DM 400 nm; BA 420 nm; Nikon eclipse 80i; Nikon Corp., Japan).

\section{Statistical Analysis}

Aphid preferences for different-aged leaves and different artificial diets were analyzed by paired $t$-test. Levene's and Kolmogorov-Smirnov tests were used to test homogeneity of the variances and normality of the data for aphid weight, EPG results, individual amino acid concentration in the leaves, total amino acid concentration in EDTA exudate, amino acid:sugar ratio in the phloem sap, glucosinolate concentration and honeydew weight. Any data that did not meet these tests were transformed using $\ln (1+\mathrm{x})$. The effects of leaf age on these factors were then analyzed using one-way analysis of variance (ANOVA), followed by Fisher's least significant difference (LSD) tests, at a significance level of $P<0.05$. All statistical analyses were performed using 
the IBM SPSS Statistics package 19 (SPSS Inc., Chicago, IL, USA).

\section{RESULTS}

\section{Myzus persicae Prefer and Perform Better on Young Cabbage Leaves}

Significantly more aphids fed on young leaves than mature leaves after $3 \mathrm{~h}(t=4.741$, $\mathrm{df}=9, P=0.001)$ and $8 \mathrm{~h}(t=5.063, \mathrm{df}=9, P=0.001$; Figure 1A $)$, while more aphids prefer to settle on young leaves compared to old leaves since $1 \mathrm{~h}$ after aphid release $(t=3.475, \mathrm{df}=9, P=0.007$; Figure 1B). By contrast, aphids distributed evenly on mature and old leaves (Figure 1C). Compared with aphids on mature or old leaves, feeding on young leaves resulted in a higher body weight $(F=18.534, \mathrm{df}=2$, 27, $P<0.001$; Figure 1D), increased nymph production $(F=5.749$, df $=2,27, P<$ 0.01 ; Figure 1E), and an increased number of adults $(F=4.408, \mathrm{df}=2,27, P<0.05$; Figure $1 \mathbf{F}$ ) at the end of the performance assay.

\section{Young Leaves Are More Nutritious}

Young cabbage leaves contained significantly higher levels of arginine $(F=17.569$, df $=2,21, P<0.001)$, serine $(F=20.829, \mathrm{df}=2,21, P<0.001)$, asparagine $(F=30.975$, $\mathrm{df}=2,21, P<0.001)$ and glutamine $(F=32.42, \mathrm{df}=2,21, P<0.001)$ than old or mature leaves (Figure 2A-B). The total amino acid content in the phloem exudate of old leaves was significantly lower than those of mature or old leaves, while total amino acid levels in mature and young phloem exudate were not statistically different $(F=12.417, \mathrm{df}=2,21, P<0.001 ;$ Figure 2D). Phloem sap amino acid:sugar molar ratio of young and old leaves was significantly higher than mature leaves $(F=15.573$, $\mathrm{df}=2,21, P<0.001 ;$ Figure 2E).

\section{Glutamine, Methionine and Valine Stimulate M. persicae Feeding}

Significantly more aphids preferred to feed on the glutamine solution after $2 \mathrm{~d}(t=$ $2.589, \mathrm{df}=9, P<0.05$; Figure 3A) or methionine solution after $1 \mathrm{~d}(t=2.632, \mathrm{df}=9$, $P<0.05$; Figure 3B) than the control, and the numbers of aphids chose glutamine solution or methionine solution increased with time. After $3 \mathrm{~d}$, aphids also showed a significant preference for the valine solution $(t=2.590, \mathrm{df}=9, P<0.05$; Figure 3C). There was no significant preference for any of the other amino acid in sucrose solution (data not shown).

\section{Young Leaves and Aphids That Feed on Them Have Higher Glucosinolate Concentrations}

Young cabbage leaves generally had higher glucosinolate contents than old or mature leaves, while old leaves contained the lowest levels (Figure 4A-B). Moreover, aphids that fed on young leaves had the highest glucosinolate levels in their bodies (Figure 4C-D), containing almost 2 times as much of the indole glucosinolates 1-methoxyindol-3-ylmethyl (1MI3M; $F=27.063$, df $=2,21, P<0.001$ ), 4-methoxyindol-3-ylmethyl (4MI3M; $F=53.271, \mathrm{df}=2,21, P<0.001)$ and indol-3-ylmethyl (I3M; $F=102.181, \mathrm{df}=2,21, P<0.001$ ) than those feeding on old 
or mature leaves (Figure 4C-D).

\section{Myzus persicae Have Longer Phloem Feeding Time and Produce More Honeydew on Young Leaves}

Aphids had a significantly longer total probing time when feeding on young leaves than on old or mature leaves $(F=4.571, \mathrm{df}=2,68, P<0.05$; Figure 5A). The mean phloem feeding duration of aphids on young leaves was about 4-6 times longer than those on old or mature leaves $(F=43.184$, $\mathrm{df}=2,68, P<0.001$; Figure 5B). Aphids fed on young leaves had approximately 2 times longer total phloem feeding time than aphids on mature or old leaves $(F=12.638, \mathrm{df}=2,68, P<0.001$; Figure 5C), while honeydew production rate was approximately 5 times higher from aphids feeding on young leaves than those on other leaves $(P<0.01$; Figure 5D).

\section{Aphid Feeding Behavior on Cabbage Leaves}

Aphids feeding on different-aged leaves had similar numbers of probes before their stylets contacting plant phloem $(F=0.896, \mathrm{df}=2,68, P=0.413$; Table 1) and spent comparable time on the first probe $(F=0.216, \mathrm{df}=2,68, P=0.806)$. Aphids had the longest phloem feeding time on young leaves, but they exhibited significantly more numbers of salivation phase $(\mathrm{E} 1)(F=20.914$, $\mathrm{df}=2,68, P<0.001)$ and phloem feeding phase (E2) $(F=21.162$, df $=2,68, P<0.001)$ when feeding on old and mature leaves. In addition, $M$. persicae infestation did not elicit callose deposits on cabbage leaves (Figure S1A), while mechanical wounding resulted in callose deposits (Figure S1B). 
TABLE 1 | Probing behavior of Myzus persicae on young, mature, and old 356 cabbage leaves.

\begin{tabular}{llll}
\hline EPG Parameters & $\begin{array}{l}\text { Young } \\
\mathbf{n = 2 5}\end{array}$ & $\begin{array}{l}\text { Mature } \\
\mathbf{n = 2 4}\end{array}$ & $\begin{array}{l}\text { Old } \\
\mathbf{n = 2 2}\end{array}$ \\
\hline $\begin{array}{l}\text { Number of probes before 1st phloem } \\
\text { phase }\end{array}$ & $7.8 \pm 1.7$ & $5.8 \pm 1.2$ & $8.5 \pm 1.6$ \\
$\begin{array}{l}\text { Time to 1st probe from start of EPG } \\
\text { (min) }\end{array}$ & $3.7 \pm 1.8 \mathbf{a}$ & $6.5 \pm 1.7 \mathbf{b}$ & $2.5 \pm 0.8 \mathbf{a b}$ \\
$\begin{array}{l}\text { Duration of 1st probe (min) } \\
\text { Time to 1st phloem phase from start } \\
\text { of EPG (h) }\end{array}$ & $99.5 \pm 31.5$ & $71.5 \pm 24.1$ & $34.9 \pm 7.3$ \\
Number of E1 & $1.7 \pm 0.2 \mathbf{a}$ & $5.0 \pm 0.6 \mathbf{b}$ & $6.0 \pm 1.0 \mathbf{b}$ \\
Number of E2 & $1.6 \pm 0.2 \mathbf{a}$ & $4.8 \pm 0.5 \mathbf{b}$ & $5.7 \pm 0.9 \mathbf{b}$ \\
$\begin{array}{l}\text { Mean duration of E1 (min) } \\
\text { Total duration of E1 (min) }\end{array}$ & $0.8 \pm 0.1$ & $1.3 \pm 0.6$ & $0.8 \pm 0.1$ \\
Total number of probes & $1.2 \pm 0.2$ & $7.4 \pm 4.1$ & $4.7 \pm 0.7$ \\
\hline
\end{tabular}

Data are means $\pm S E$ and different letters within each row indicate significant differences $(P<0.05)$. E1, salivation; E2, phloem ingestion; $n$, number of replicates. 


\section{DISCUSSION}

In this study, M. persicae exhibited a clear preference for young leaves, which is in agreement with previous findings for other aphid species (Gould et al., 2007; Cibils-Stewart et al., 2015). Aphids generally decide to settle on a host plant before their stylets reaching the phloem bundles (Powell et al., 2006). We found that $M$. persicae could distinguish young leaves from old leaves within 1 hour, but aphids spent at least 2 hours reaching the phloem, suggesting that cues that determine aphid preference locate at epidermal or mesophyll cells. Stimulatory and deterrent metabolites as well as plant cell wall composition are involved in host plant preference by aphids (Goggin, 2007). Myzus persicae showed a strong preference for the sucrose solution containing glutamine, which is present at a significant higher concentration in young leaves, suggesting that glutamine may stimulate aphid feeding and contribute to the attractiveness of young leaves for $M$. persicae. Some specialist insect herbivores rely on glucosinolates as settlement cues, which generally were present at higher levels in young leaves (Gabrys and Tjallingii, 2002; Furlong et al., 2013). However, since indole glucosinolates can act as deterrents to the generalist aphid $M$. persicae, this is unlikely to explain aphid preference for young leaves (Kim and Jander, 2007). Finally, plant cells of young leaves are at expanding stage and have flexible cell walls that may exert lower restriction to aphid stylet penetration (Divol et al., 2007).

Aphids continuously feed on plants, removing large amounts of phloem sap and reducing the hydrostatic pressure of phloem, which alters the source-sink patterns within the host plant (Girousse et al., 2005; Züst and Agrawal, 2016). According to Münch (1930), source tissues such as mature and old leaves have higher hydrostatic pressure than sink tissues such as young leaves and reproductive organs, and so it would be expected that aphids would ingest more phloem sap by feeding on source tissues. However, we found that $M$. persicae produced approximately 5 times as much honeydew per hour when they fed on young leaves, likely due to these leaves being strong natural sinks of plants that can easily draw phloem sap from source tissues during aphid feeding. The EPG results also indicated that $M$. persicae spent approximately 2 times longer feeding from phloem of young leaves than mature or old leaves. Moreover, shorter mean phloem feeding time of aphids on mature and old leaves indicated that aphids frequently withdraw their stylets from phloem of these leaves which is possibly due to reduced phloem supply caused by the depletion of phloem sap. Since $M$. persicae infestation elicits no visible callose deposits, this is not possible due to callose block of the phloem.

Amino acids are the major nutrition for aphids and are a key limiting factor for aphid growth, whose concentration and composition can influence aphid performance 
(Douglas, 2003). The amino acid:sugar molar ratio in the phloem sap was about 5 times higher in young leaves than in mature leaves, indicating a higher nutrition quality in phloem of young leaves. Although old leaves had a similar amino acid:sugar molar ratio in their phloem sap to young leaves, aphids obtained only one-fifth amount of phloem sap on these leaves. The higher amino acid:sugar ratio in phloem sap of old leaves may be due to the degradation of protein caused by leaf senescence (Lim et al., 2007). Reduced phloem sap ingestion is usually involved in plant resistance to aphids, suggesting the significance of phloem sap availability in determining aphid performance (Will et al., 2013). These results suggest that the amount of nutrition that aphids can obtained is as important as nutrition quality. Future research on plant-aphid interaction should pay more attention to phloem sap availability.

We found that young cabbage leaves contained the highest levels of glucosinolates, supporting the previous findings of Lambdon et al. (2003). Several studies have shown that glucosinolates are involved in plant resistance to aphids, despite aphids rarely contacting myrosinase during feeding (Kim et al., 2008; Pfalz et al., 2009). However, in our earlier study, we found that M. persicae infested leaves had high contents of indole glucosinolates but aphids grew better on these leaves (Cao et al., 2016). Similarly, the cabbage aphid, Brevicoryne brassicae L., also performs better when feeding on reproductive tissues (flowering canopy) than on vegetative tissues (leaves), despite the flowering canopy having higher levels of glucosinolates (Cibils-Stewart et al., 2015). And in this study, M. persicae grew better on young leaves that contained higher levels of glucosinolates. These findings suggest that the effects of glucosinolates in plant resistance to aphids has significant variation, which may be due to nutrition compensate or aphid adaption. and aphids tend to feed on more nutritious tissues to maximize their fitness, regardless of the higher levels of toxics in these tissues. In addition, aphids generally had higher levels of I3M and $1 \mathrm{MI} 3 \mathrm{M}$ in their bodies than leaves they were feeding on, but aphids feeding on young leaves contained significantly lower 4MI3M, a more toxic indole glucosinolates for $M$. persicae, than that in young leaves, implying that aphids may have converted this glucosinolates to lesser toxic metabolites.

Both toxic metabolites and nutrition are essential factors in plant resistance to aphids, but aphids have evolved strategies to cope with these constraints by detoxifying the toxics and selecting feeding sites (Dreyer and Campbell, 1987; Mathers et al., 2016). We found that young cabbage leaves contained higher levels of glucosinolates, but also provide more nutrition, contributing to the improved performance of $M$. persicae. The source-sink relationship within plants influences phloem sap availability and thus aphid performance, which deserves more attention in the research of aphid-plant interaction.

\section{AUTHOR CONTRIBUTIONS}

$\mathrm{HC}$ originally formulated the idea and designed the experiments. $\mathrm{HC}$ and $\mathrm{ZZ}$ performed the experiments. HC did the statistical analyses and wrote the manuscript. $\mathrm{XW}$ and TL provided editorial advice. TL provided funding for the project. 


\section{FUNDING}

This work was supported by the National Basic Research Program of Ministry of Science and Technology, China (973 Program, 2013CB127600), Natural Science Foundation of China (31272089), Special Fund for Agro-Scientific Research in the Public Interest (201103022), and China Agriculture Research System (CARS-25-B-06).

\section{ACKNOWLEDGMENTS}

We are grateful for the assistance of all the members in the Key Laboratory of Applied Entomology, Northwest A\&F University, Yangling, Shaanxi, China.

\section{REFERENCES}

Barton, K.E., and Koricheva, J. (2010) The ontogeny of plant defense and herbivory: characterizing general patterns using meta-analysis. Am. Nat. 175, 481-493. doi:10.1086/650722

Cao, H.H., Liu, H.R., Zhang, Z.F., and Liu, T.X. (2016) The green peach aphid Myzus persicae perform better on pre-infested Chinese cabbage Brassica pekinensis by enhancing host plant nutritional quality. Sci. Rep. 6:21954. doi:10.1038/srep21954

Cao, H.H., Zhang, M., Zhao, H., Zhang, Y., Wang, X.X., Guo, SS, et al. (2014a) Deciphering the mechanism of beta-aminobutyric acid-induced resistance in wheat to the grain aphid, Sitobion avenae. PLoS One 9: e91768. doi:10.1371/journal.pone.0091768

Cao, H.H., Wang, S.H., and Liu, T.X. (2014b) Jasmonate- and salicylate-induced defenses in wheat affect host preference and probing behavior but not performance of the grain aphid, Sitobion avenae. Insect Sci. 21, 47-55. doi:10.1111/1744-7917.12023

Cibils-Stewart, X., Sandercock, B.K., and McCornack, B.P. (2015) Feeding location affects demographic performance of cabbage aphids on winter canola. Entomol. Exp. Appl. 156, 149-159. doi:10.1111/eea.12325

Cole, R.A. (1997). The relative importance of glucosinolates and amino acids to the development of two aphid pests Brevicoryne brassicae and Myzus persicae on wild and cultivated brassica species. Entomol. Exp. Appl. 85, 121-133. doi:10.1046/j.1570-7458.1997.00242.x

Divol, F., Vilaine, F., Thibivilliers, S., Kusiak, C., Sauge, M.H., and Dinant, S. (2007) Involvement of the xyloglucan endotransglycosylase/hydrolases encoded by celery XTH1 and Arabidopsis XTH33 in the phloem response to aphids. Plant Cell Environ. 30, 187-201. doi:10.1111/j.1365-3040.2006.01618.x

Douglas, A.E. (2003) The nutritional physiology of aphids. Adv. Insect Physiol. 31, 73-140. doi:10.1016/s0065-2806(03)31002-1

Dreyer, D.L., and Campbell, B.C. (1987) Chemical basis of host-plant resistance to aphids. Plant Cell Environ. 10, 353-361. doi:10.1111/1365-3040.ep11603601

Furlong, M.J., Wright, D.J., and Dosdall, L.M. (2013) Diamondback moth ecology and management: problems, progress, and prospects. Annu. Rev. Entomol. 58, 
517-541. doi:10.1146/annurev-ento-120811-153605

Gabrys, B., and Tjallingii, W. (2002) The role of sinigrin in host plant recognition by aphids during initial plant penetration. Entomol. Exp. Appl.104, 89-93. doi: 10.1046/j.1570-7458.2002.00994.x

Girousse, C., Moulia, B., Silk, W., and Bonnemain, J.L. (2005) Aphid infestation causes different changes in carbon and nitrogen allocation in alfalfa stems as well as different inhibitions of longitudinal and radial expansion. Plant Physiol. 137, 1474-1484. doi:10.1104/pp.104.057430

Goggin, F.L. (2007) Plant-aphid interactions: molecular and ecological perspectives. Curr. Opin. Plant Biol. 10, 399-408. doi:10.1016/j.pbi.2007.06.004

Gould, G.G., Jones, C.G., Rifleman, P., Perez, A., and Coleman, J.S. (2007) Variation in eastern cottonwood (Populus deltoides Bartr.) phloem sap content caused by leaf development may affect feeding site selection behavior of the aphid, Chaitophorous populicola Thomas (Homoptera: Aphididae). Environ. Entomol. 36, 1212-1225. doi:10.1603/0046-225x(2007)36[1212:viecpd]2.0.co;2

Hopkins, R.J., Van Dam, N.M., and Van Loon, J.J. (2009). Role of glucosinolates in insect-plant relationships and multitrophic interactions. Anпи. Rev. Entomol. 54, 57-83. doi: 10.1146/annurev.ento.54.110807.090623.

Kim, J.H., and Jander, G. (2007) Myzus persicae (green peach aphid) feeding on Arabidopsis induces the formation of a deterrent indole glucosinolate. Plant $J$. 49, 1008-1019. doi:10.1111/j.1365-313X.2006.03019.x

Kim, J.H., Lee, B.W., Schroeder, F.C., and Jander, G. (2008) Identification of indole glucosinolate breakdown products with antifeedant effects on Myzus persicae (green peach aphid). Plant J. 54, 1015-1026. doi:10.1111/j.1365-313X.2008.03476.x

Kohler, A., Maag, D., Veyrat, N., Glauser, G., Wolfender, J.L., Turlings, T.C., and Erb, M. (2015) Within-plant distribution of 1,4-benzoxazin-3-ones contributes to herbivore niche differentiation in maize. Plant Cell Environ. 38, 1081-1093. doi:10.1111/pce.12464

Lambdon, P.W., Hassall, M., Boar, R.R., and Mithen, R. (2003) Asynchrony in the nitrogen and glucosinolate leaf-age profiles of Brassica: is this a defensive strategy against generalist herbivores? Agric. Ecosyst. Environ. 97, 205-214. doi:10.1016/s0167-8809(03)00036-7

Larson, K.C., and Whitham, T.G. (1991) Manipulation of food resources by a gall-forming aphid: the physiology of sink-source interactions. Oecologia 88 , 15-21. doi:10.1007/BF00328398

Lim, P.O., Kim, H.J., and Nam, H.G. (2007). Leaf Senescence. Annu. Rev. Plant Biol. 58, 115-136. doi:10.1146/annurev.arplant.57.032905.105316

Mathers, T.C., Chen, Y., Kaithakottil, G., Legeai, F., Mugford, S.T., Baa-Puyoulet, P., et al. (2016). A clonally reproducing generalist aphid pest colonises diverse host plants by rapid transcriptional plasticity of duplicated gene clusters. bioRxiv, 063610. doi: http://dx.doi.org/10.1101/063610

Münch, E. (1930) Die Stoffbewegunen in der Pflanze. Jena: Verlag von Gustav Fischer, 15, 488-489 
Nishimura, M.T., Stein, M., Hou, B.H., Vogel, J.P., Edwards, H., and Somerville, S.C. (2003) Loss of a callose synthase results in salicylic acid-dependent disease resistance. Science 301, 969-972. doi:10.1126/science.1086716

Pfalz, M., Vogel, H., and Kroymann, J. (2009) The gene controlling the indole glucosinolate modifier1 quantitative trait locus alters indole glucosinolate structures and aphid resistance in Arabidopsis. Plant Cell 21, 985-999. doi: http://dx.doi.org/10.1105/tpc.108.063115

Powell, G., Tosh, C.R., and Hardie, J. (2006) Host plant selection by aphids: behavioral, evolutionary, and applied perspectives. Annu. Rev. Entomol. 51, 309-330. doi:10.1146/annurev.ento.51.110104.151107

Prado, E., and Tjallingii, W.F. (1994) Aphid activities during sieve element punctures. Entomol. Exp. Appl. 72, 157-165. doi: 10.1111/j.1570-7458.1994.tb01813.x

Rochfort, S.J., Trenerry, V.C., Imsic, M., Panozzo, J., and Jones, R. (2008) Class targeted metabolomics: ESI ion trap screening methods for glucosinolates based on $\mathrm{MS}^{\mathrm{n}}$ fragmentation. Phytochemistry 69, 1671-1679. doi:10.1016/j.phytochem.2008.02.010

Sarria, E., Cid, M., Garzo, E., and Fereres, A. (2009) Excel Workbook for automatic parameter calculation of EPG data. Comput. Electron. Agr. 67, 35-42. doi:10.1016/j.compag.2009.02.006

Schuman, M.C., and Baldwin, I.T. (2016) The layers of plant responses to insect herbivores. Annu. Rev. Entomol. 61, 373-394. doi:10.1146/annurev-ento-010715-023851

Thiele, B., Fullner, K., Stein, N., Oldiges, M., Kuhn, A.J., and Hofmann, D. (2008) Analysis of amino acids without derivatization in barley extracts by LC-MS-MS. Anal. Bioanal. Chem. 391, 2663-2672. doi:10.1007/s00216-008-2167-9

Will, T., Furch, A.C.U., and Zimmermann, M.R. (2013) How phloem-feeding insects face the challenge of phloem-located defenses. Front. Plant Sci. 4, doi:10.3389/fpls.2013.00336

Züst, T., and Agrawal, A.A. (2016). Mechanisms and evolution of plant resistance to aphids. Nature Plants 2, 15206. doi: 10.1038/nplants.2015.206.

Conflict of Interest Statement: The authors declare that the research was conducted in the absence of any commercial or financial relationships that could be construed as a potential conflict of interest.

Figure legends

FIGURE 1 | Preference and performance of Myzus persicae on different-aged cabbage leaves. Preference of $M$. persicae for pairs of different-aged leaves (A-C) (paired $t$-test: $* P<0.05$ ); and performance of $M$. persicae in terms of body weight (D), nymph production (E), and number of adults (F). Different letters above the bars indicate significant differences $(P<0.05)$. Values are means $\pm \operatorname{SE}(\mathrm{n}=10)$. 
600

FIGURE 2 | Nutrition quality in cabbage leaves for Myzus persicae. Amino acid contents in different-aged leaves (A-C), and the amino acid concentrations (D) and amino acid:sugar molar ratios (E) in the phloem sap. Different letters above the bars indicate significant differences $(P<0.05)$. Values are means $\pm \operatorname{SE}(n=8)$.

FIGURE 3 | Myzus persicae preference for glutamine, methionine, and valine. Number of adult $M$. persicae selecting a $15 \%$ sucrose solution (control) or a sucrose solution containing glutamine (A), methionine (B), or valine $(\mathbf{C})$. (Paired $t$-test: $* P<$ $0.05, * * P<0.01)$. Values are means $\pm \mathrm{SE}(\mathrm{n}=10)$.

FIGURE 4 | Concentrations of glucosinolates in different-aged cabbage leaves and aphids feed on them. Glucosinolates concentrations in different-aged cabbage leaves (A-B), and in Myzus persicae feeding on these leaves (C-D). Different letters above the bars indicate significant differences $(P<0.05)$. Values are means $\pm \mathrm{SE}(\mathrm{n}=$ 8). Glucosinolate side chain abbreviations: 4MTB, 4-methylsulfinylbutyl; I3M, indol-3-ylmethyl; 4MI3M, 4-methoxyindol-3-ylmethyl; 4OHI3M, 4-hydroxyindol-3-ylmethyl; 1MI3M, 1-methoxyindol-3-ylmethyl; 4MSB, 4-Methylsuphinylbutyl.

FIGURE 5 | Myzus persicae phloem feeding activities and honeydew production rate on different-aged cabbage leaves. Total probing time (A), mean phloem feeding duration (B), total phloem feeding duration (C), and honeydew production (D) by $M$. persicae on cabbage leaves. Different letters above the bars indicate significant differences $(P<0.05)$. Values are means $\pm \operatorname{SE}(n=22-25$ for $A-C ; n=5$ for $\mathrm{D})$.

FIGURE 6 | Model depicting cabbage-Myzus persicae interaction. Physical and chemical cues from the peripheral (nonvascular) cells control aphid preference and aphids only decide to settle or leave after penetrating the plant tissue (Powell et al., 2006). In young leaves, the higher concentration of glutamine is likely involved in host preference of $M$. persicae, while the high contents of glucosinolates act as feeding deterrents (Kim et al., 2008). Aphids are external sinks of plants and so can obtain more nutrition when feeding on young leaves, which are strong natural sinks of plants.

Red arrows indicate phloem flow direction. 

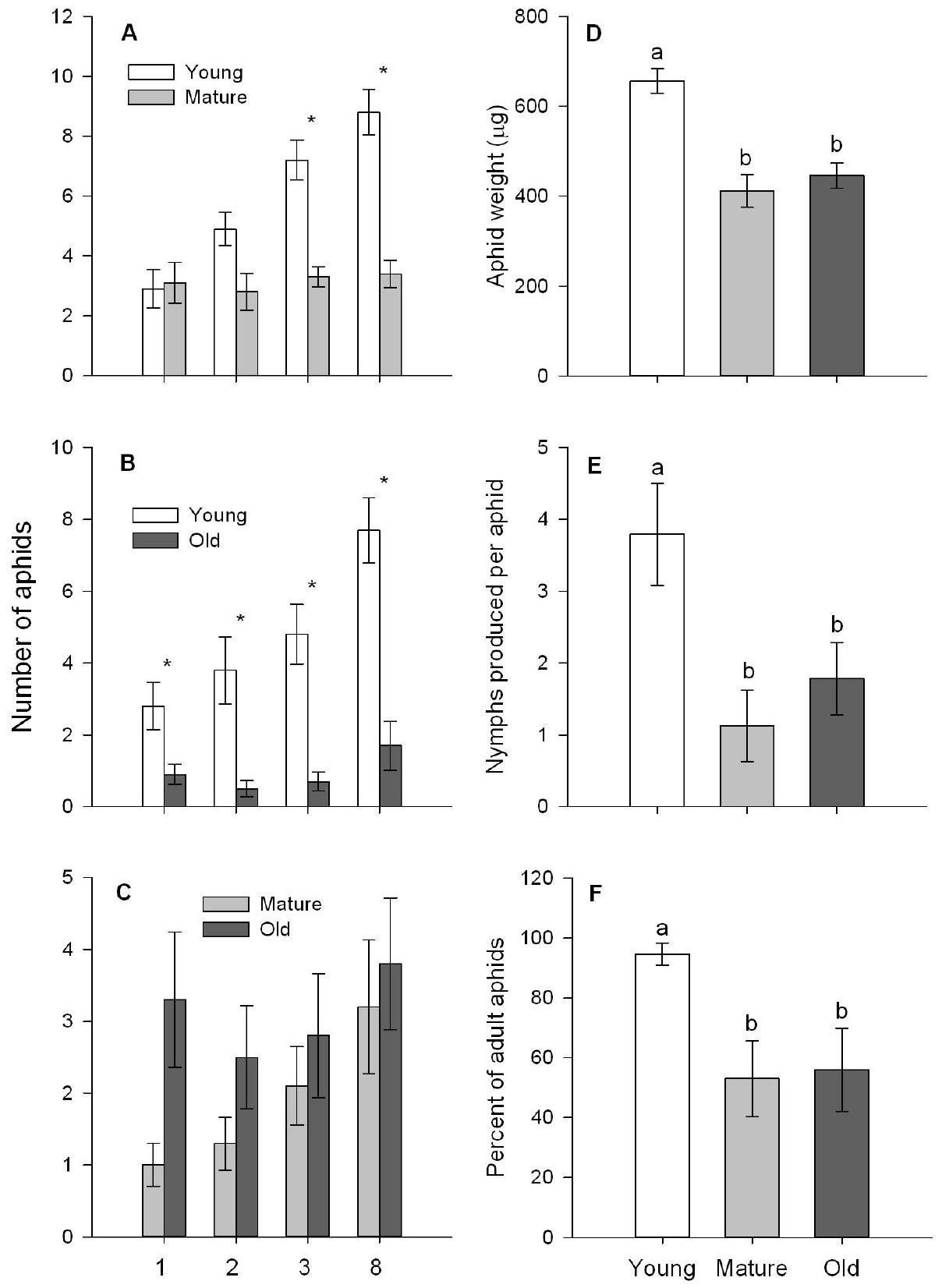

Time after aphid release 

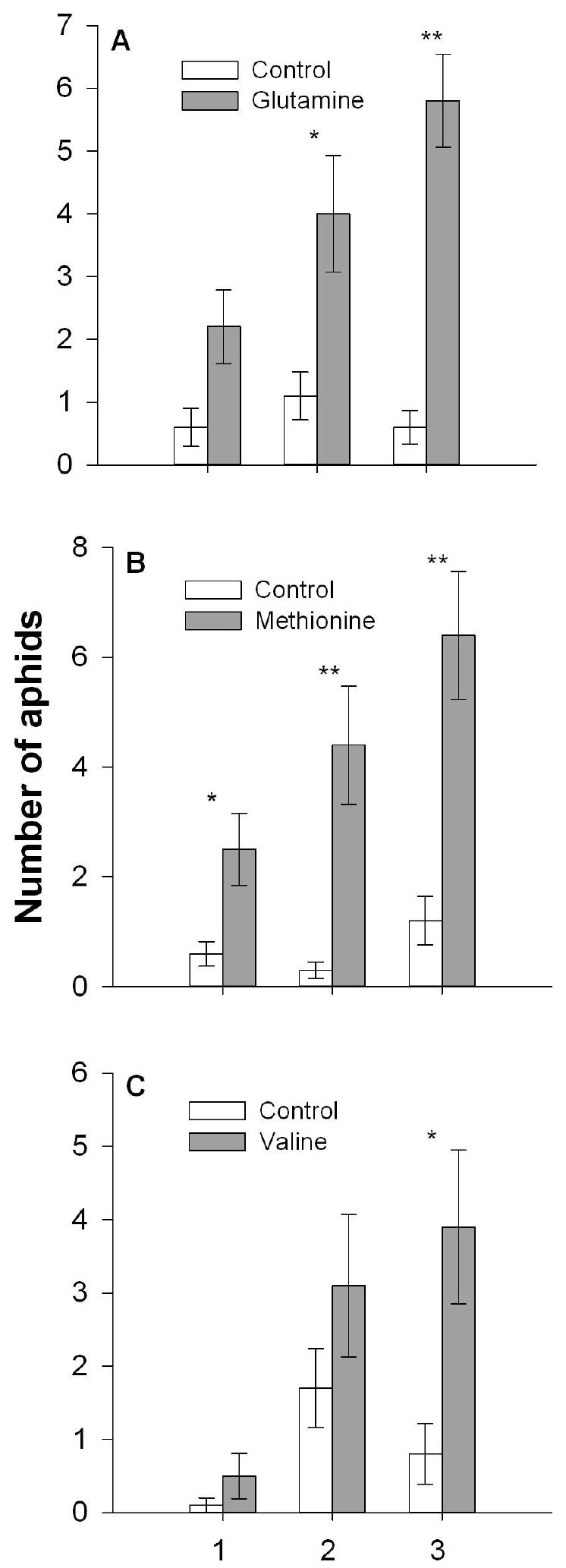

Days after aphid release 

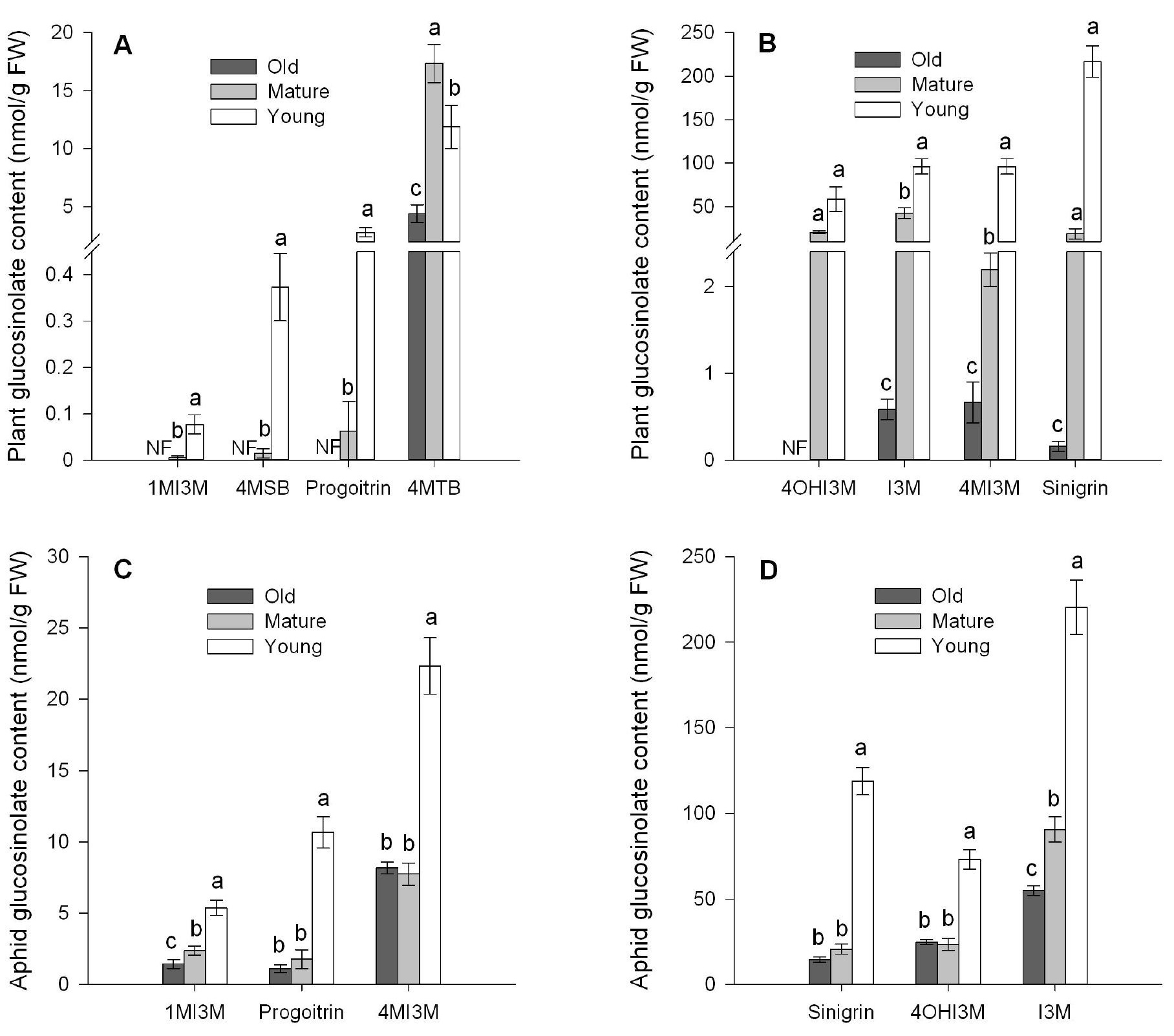

Glucosinolates 

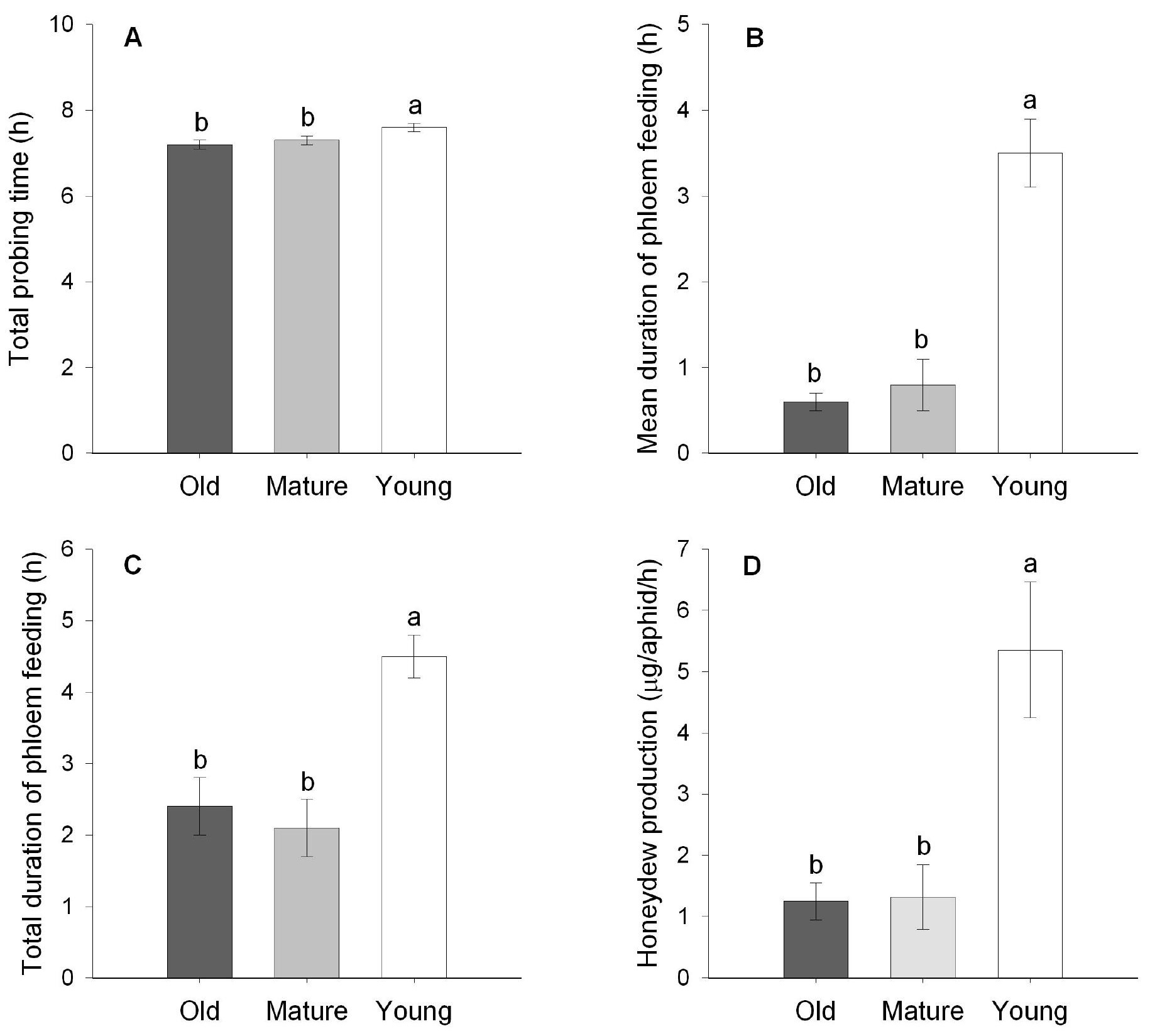


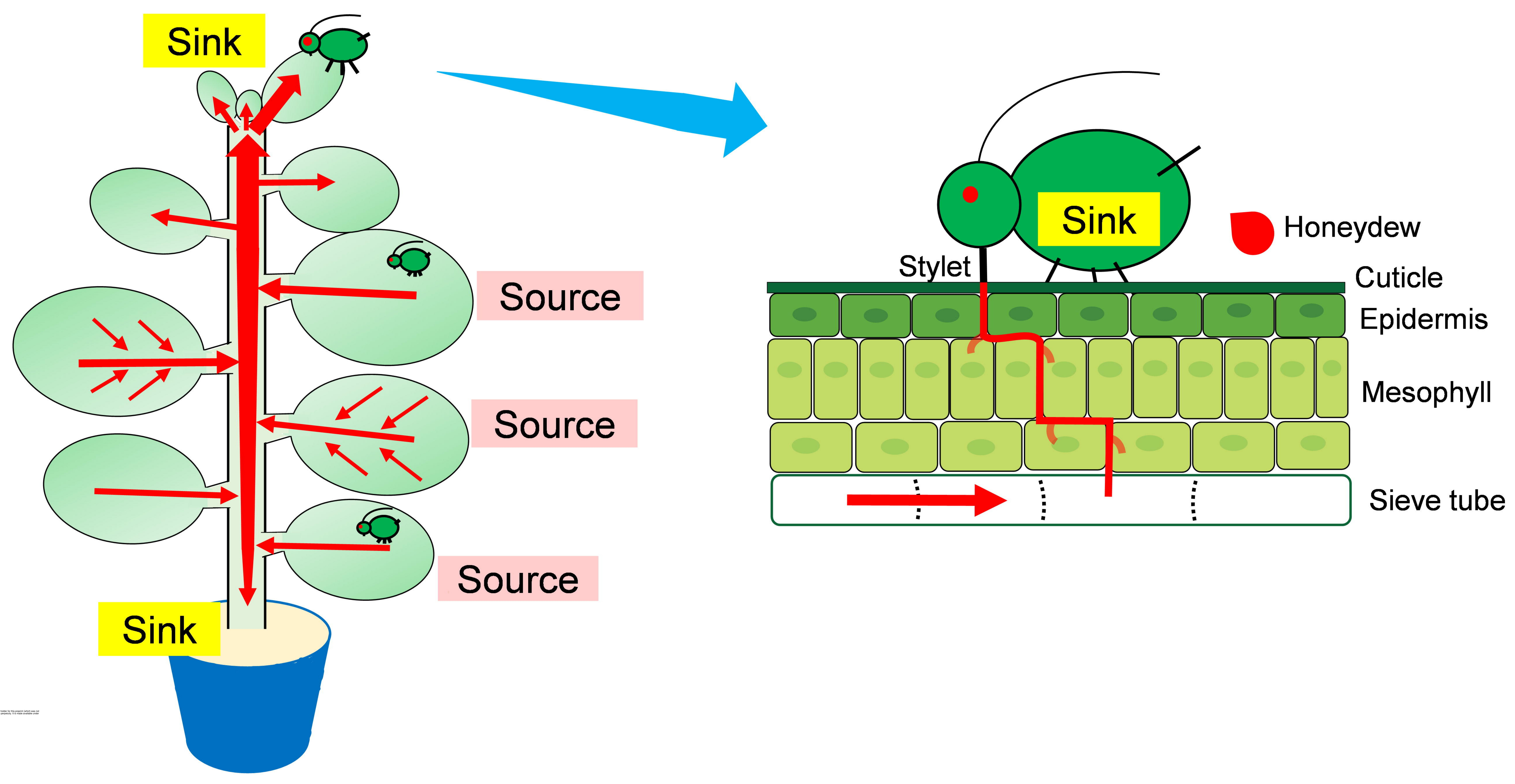

